well suited to the measurement of the ratio, $R$, of the intensity of $N_{1}+N_{2}$ to that of $H \beta$. This is because the ratio is often large, the sensitivity of the emulsion is changing rapidly in the spectral region involved, and the nebular images in the light of hydrogen and [OIII] often show structural differences. Accordingly, a cheek upon the photographic determinations of this ratio is very much to be desired.

Between May and August 1953 we obtained some photoelectric measures of the ratio $R$ in a number of planetary nebulæ using the 24-36-in. Schmidt telescope of the Warner and Swasey Observatory. The objective prism provided a spectral image of each nebula at the focus $\left(97^{\prime \prime} / \mathrm{mm}\right.$. and $275 \mathrm{~A} . / \mathrm{mm}$. at $H \beta)$. An $R C A$ type $1 P 21$ photomultiplier tube with associated equipment ${ }^{2}$ measured directly the total light intensity in the various spectral images. Details of the procedure for eliminating the effects of overlapping images, spectral sensitivity of the cell, atmospheric extinction, and the stellar continuum will be published. elsewhere.

For the five planetaries listed in the first column of Table 1 numerical values for the ratio, $R$, are given in the second column. The probable errors of $R$ are listed in the third column, and the number of runs over the spectrum of the nebula, $n$, are given in the fourth. The more precise photoelectric results confirm the accuracy of Aller's photographic measures ${ }^{3}$ given in the last column.

\begin{tabular}{|c|c|c|c|c|}
\multicolumn{2}{c|}{ Table 1 } \\
\hline NGC & $\begin{array}{c}R \\
\text { photoelectric }\end{array}$ & $\begin{array}{c}\text { Probable } \\
\text { error }\end{array}$ & $n$ & $\begin{array}{c}\boldsymbol{R} \\
\text { photographic }\end{array}$ \\
\hline $\mathbf{6 , 5 4 3}$ & $8 \cdot 07$ & $0 \cdot 14$ & 10 & $8 \cdot 1$ \\
6,826 & $9 \cdot 8$ & $0 \cdot 2$ & 3 & $10 \cdot 7$ \\
6,572 & $15 \cdot 75$ & $0 \cdot 13$ & 11 & $12 \cdot 9$ \\
7,027 & 20 & 1 & $18 \cdot 7$ \\
7,662 & $20 \cdot 0$ & $1 \cdot 4$ & 4 & $21 \cdot 2$ \\
\hline
\end{tabular}

The photoelectric observations provide additional information which is very difficult to obtain by other methods, namely, the intensity of the emission lines in terms of the energy in the continuum of the central star. Results for two planetaries are given in Table 2. For each, the number in the second column is the intensity of $N_{1}$ and $N_{2}$ combined in terms of the energy per angstrom in the stellar continuum at $\lambda 5,007$.

Table 2

\begin{tabular}{|c|c|c|c|}
\hline$N G C$ & $N_{1}+N_{2} /$ cont. & Probable error & $n$ \\
\hline 6,826 & 2,300 & 140 & 3 \\
7,662 & 1,500 & 60 & 4 \\
\hline
\end{tabular}

We wish to thank Mr. L. H. Aller for directing our attention to this problem from the observational point of view.

David Dunlap Observatory,

University of Toronto,

Richmond Hill, Ontario.

Donald A. MaCRaE

JuRgen Stock

Warner and Swasey Observatory,

Case Institute of Technology,

East Cleveland, Ohio. Nov. 30.

${ }^{1}$ See, for example, Menzel, D. H., Aller, L. H., and Hebb, M. H., Astrophys. J., 93,230 (1941), New values for the target areas have been
$400(1953)$.

2 MacRae, D. A., Astro. J., 58, 43 (1953).

${ }^{3}$ Astrophy's. J., 93, 236 (1941); 113, 125 (1951).

\section{Fermi's Thermodynamic Theory of the Production of Pions}

Fermi's ${ }^{1}$ statistical thermodynamic theory of the generation of pions in collisions of high-energy nucleons has stimulated considerable discussion of the problem. The theory is in reasonably good agreement with observation; but the theory is not free, as Fermi himself and others ${ }^{2}$ have pointed out, from conceptional difficulties. However, the purpose of the present note is to show that in the usual deriva. tion $^{1}$ for the number of pions produced on Fermi's theory, the contribution of the pions in the lowest energy-state is not adequately taken into account. In fact, because of the Lorentz-contraction of the volume (in which pions are produced) the usual estimate of the total number of pions produced has an appreciable error. In the absence of the Lorentzcontraction the error would be entirely negligible.

Let us consider the sum (in the usual notation) :

$$
N_{1}=\sum_{i} \frac{1}{e^{\beta t_{i}}-1}, \beta=1 / k T \text {. }
$$

Let $N$ stand for the integral

$$
N=\frac{4 \pi g \Omega}{(2 \pi c \hbar)^{3}} \int_{0}^{\infty} \frac{t^{2} d t}{e^{\beta t}-1}=\frac{4 \pi g \Omega}{(2 \pi c \cdot)^{3}} 2 ! \frac{\zeta(3)}{\beta^{3}},
$$

where $\Omega$ is the volume of the assembly and $g$ the weight factor for the pions $(g=3)$.

If we write

$$
N_{1}=N+N_{0} \text {, }
$$

then it follows that

$$
N_{0}=\alpha g k T / \mu c^{2},
$$

where $\alpha$ is a numerical factor of the order unity. If $W$ is the total energy of the colliding nucleons in the co-ordinate system (called $C$-system) in which the total momentum is zero, we have, on substituting for $(k T)$ in terms of $W$ :

$$
\frac{N_{0}}{N}=\frac{\alpha[9 \pi \zeta(4)]^{1 / 2}}{2 \zeta(3)}\left(\frac{g \mu}{M}\right)^{1 / 2} \frac{1}{(\gamma f)^{1 / 2}},
$$

where $M$ is nucleon rest mass, $\gamma=W / M c^{2}$, and $f$ is defined by

$$
f=\Omega / \Omega_{0}, \Omega_{0}=\frac{4 \pi}{3}(\hbar / \mu c)^{3} .
$$

When $f \sim 1, \frac{N}{N}^{0} \rightarrow 0$ for large $\gamma$. However, in Fermi's theory the volume available in the $C$-system for production of pions is not $\Omega_{0}$, but the Lorentzcontracted volume $2 \Omega_{0} / \gamma$. Hence, we have

$$
\frac{N_{0}}{N}=\alpha \frac{[9 \pi \zeta(4)]^{1 / 2}}{2 \zeta(3)}-\left(\frac{g \mu}{2 M}\right)^{1 / 2} \sim 0.54 \text { (for } \alpha=\frac{1}{2} \text { ). }
$$

Thus the number $N_{0}$ is of the same order as $N$, and hence Fermi's expression (equation (2)) seriously underestimates the total number of pions produced. In the laboratory system the $N_{0}$ pions will form a narrow jet moving along the direction of the incident nucleon, each pion having an energy $\gamma \mu c^{2} / 2$.

Department of Physics, D. S. KOTHARI University of Delhi.

$$
\text { Dec. } 1 .
$$

${ }^{1}$ Fermi, E., Prog. Theor. Phys., 5, 570 (1950) ; Phys. Rev., 81, 683 (1951).

${ }^{3}$ Bhabha, H. J., Proc. Roy. Soc., A, 219, 293 (1953). Auluck, F. C., and Kothari, D. S., Phys. Rev., 90, 1002 (1953). 